\section{Autocontouring Versus Manual Contouring}

TO THE EDITOR: We read with interest the study of Wu et al. (1) regarding autocontouring methodologies for target delineation in PET/CT for non-small cell lung cancer (NSCLC). Seventeen NSCLC tumors were delineated with both automated and manual approaches, using either combined PET/CT or CT and PET independently. As expected, manual contouring of PET uptake correlated better with the maximum diameter of the primary tumor than did autocontouring using a fixed threshold at $50 \%$ of maximum tumor uptake. We believe that this result is largely associated with the various shortcomings of fixed-threshold approaches, a point that needs to be clearly emphasized.

The authors have previously demonstrated that the best correlation between histopathology-derived maximum tumor diameters and image-derived ones was obtained using a 50\% fixed threshold (2). This conclusion was reached by comparison with results obtained using other fixed-threshold values (from 20\% to $55 \%$ ), with a modest correlation of 0.77 and nonstatistically significant differences from the other fixed-threshold values tested. Most significantly, the use of a 50\% fixed threshold led to differences larger than $1 \mathrm{~cm}$ in half the tumors considered. Such differences in maximum tumor diameter will most certainly lead to larger differences in the overall 3-dimensional volume. Considering similar comparisons based on 3-dimensional NSCLC tumor volumes determined by histopathology, other authors have demonstrated that an "optimal" threshold cannot be determined; considerable variability is seen $(20 \%-42 \%[31 \% \pm 11 \%]$ of the maximum), whereas CT-based volumes significantly overestimated the pathologic volume (3).

It is therefore important to emphasize that a fixed threshold (irrespective of its absolute value) is not an adequate methodology to delineate elevated uptake signal in PET, because of its binary, deterministic nature and lack of robustness versus varying contrast and noise conditions $(4,5)$. To account for these widely documented literature findings concerning tumor target delineation incorporating PET uptake information, fixed thresholding should be avoided, and at the very least, methodologies considering target-to-background ratios such as adaptive thresholding $(5,6)$ should be favored. Eventually, the wider availability of automatic segmentation approaches (7-10), some of which can account for the presence of heterogeneous tumor uptake (7), may improve the accuracy and reproducibility of adaptive thresholding (11) for determination of functional tumor volume.

Considering all these facts, we do agree with the authors that manual contouring should be preferred to autocontouring at a 50\% threshold for functional tumor volume delineation. On the other hand, one should consider that manual delineation of PET uptake is not the ideal approach either, for multiple reasons. Most importantly, it represents a long process, particularly when it has to be performed in 3 dimensions, and it is inherently of low reproducibility (11).

We therefore recommend that future studies investigating this issue include the use of advanced image segmentation approaches (4-10), which have demonstrated improved performance in com-

COPYRIGHT @ 2011 by the Society of Nuclear Medicine, Inc. parison to a fixed threshold and may therefore lead to alternative or complementary conclusions regarding the role of manual contouring. Irrespective of the performance of a segmentation algorithm, operator intervention will always be necessary to appropriately identify the functional uptake of interest and avoid the inclusion of non-tumor-specific uptake.

\section{REFERENCES}

1. Wu K, Ung YC, Hwang D, et al. Autocontouring and manual contouring: which is the better method for target delineation using ${ }^{18} \mathrm{~F}-\mathrm{FDG}$ PET/CT in non-small cell lung cancer? J Nucl Med. 2010;51:1517-1523.

2. Wu K, Ung YC, Hornby J, et al. PET CT thresholds for radiotherapy target definition in non-small-cell lung cancer: how close are we to the pathologic findings? Int J Radiat Oncol Biol Phys. 2010;77:699-706.

3. Yu J, Li X, Xing L, et al. Comparison of tumor volumes as determined by pathologic examination and FDG-PET/CT images of non-small-cell lung cancer: a pilot study. Int J Radiat Oncol Biol Phys. 2009;75:1468-1474.

4. Hatt M, Turzo A, Roux C, et al. A fuzzy Bayesian locally adaptive segmentation approach for volume determination in PET. IEEE Trans Med Imaging. 2009;28:881-893.

5. Nestle U, Kremp S, Schaefer-Schuler A, et al. Comparison of different methods for delineation of ${ }^{18} \mathrm{~F}$-FDG PET-positive tissue for target volume definition in radiotherapy of patients with non-small cell lung cancer. J Nucl Med. 2005;46: 1342-1348.

6. Daisne JF, Sibomana M, Bol A, et al. Tri-dimensional automatic segmentation of PET volumes based on measured source-to-background ratios: influence of reconstruction algorithms. Radiother Oncol. 2003;69:247-250.

7. Hatt M, Cheze le Rest C, Descourt P, et al. Accurate automatic delineation of heterogeneous functional volumes in positron emission tomography for oncology applications. Int J Radiat Oncol Biol Phys. 2010;77:301-308.

8. Geets X, Lee JA, Bol A, et al. A gradient-based method for segmenting FDGPET images: methodology and validation. Eur J Nucl Med Mol Imaging. 2007;34:1427-1438.

9. Montgomery DWG, Amira A, Zaidi H. Fully automated segmentation of oncological PET volumes using a combined multiscale and statistical model. Med Phys. 2007;34:722-736.

10. Yu H, Caldwell C, Mah K, et al. Automated radiation targeting in head-andneck cancer using region-based texture analysis of PET and CT images? Int $J$ Radiat Oncol Biol Phys. 2009;75:618-625.

11. Hatt M, Cheze Le Rest C, Aboagye EO, et al. Reproducibility of ${ }^{18} \mathrm{~F}-\mathrm{FDG}$ and ${ }^{18}$ F-FLT PET tumor volume measurements. J Nucl Med. 2010;51:1368-1376.

\section{Mathieu Hatt* \\ Dimitris Visvikis \\ Catherine Cheze Le Rest \\ *INSERM U650 LaTIM \\ Batînent 2bis (I3S), CHU Morvan, \\ 5 Avenue Foch, Brest 29609, France \\ E-mail: hatt@univ-brest.fr}

DOI: 10.2967/jnumed.110.084897

REPLY: We thank Dr. Hatt and colleagues for their interest in and comments about our study of autocontouring and manual contouring for target delineation using ${ }^{18} \mathrm{~F}-\mathrm{FDG} \mathrm{PET} / \mathrm{CT}$ in nonsmall cell lung cancer (NSCLC) (1). These authors are extremely accomplished in the use of PET/CT in NSCLC. We think their statement that a fixed threshold is not an adequate methodology because of its considerable variability is reasonable. 
There are limited data on contouring the gross tumor volume (GTV) using PET/CT thresholds correlated with tumor size on histopathologic examination. Our study demonstrated that using the $50 \%$ fixed threshold for contouring GTV produced the best correlation between maximum tumor diameters and histopathologic findings (2). However, the $50 \%$ fixed threshold led to a larger difference in the diameter of GTV on PET, and CT-based volume significantly overestimated the pathologic volume. In fact, the window and level of CT also led to more differences in determining the CT-based volume (2). Much uncertainty exists regarding the most appropriate threshold to define a PET target volume in NSCLC radiation treatment planning. The use of a standardized uptake value (SUV) fixedthreshold intensity to define a tumor on PET may be inadequate for target volume definition and tends to underestimate target volumes (3). Nestle et al. (4) demonstrated that a GTV applying a threshold of $40 \%$ of the maximum SUV does not appear to be suitable for target volume delineation, although they used CT volume compared with PET volume because there was no available pathology correlation. For laryngeal tumors, the segmented volumes by the gradient-based method agreed with those delineated on the macroscopic specimens, whereas the threshold-based method overestimated the true volume by $68 \%$ (5). Yu et al. (6) have shown that the absolute SUV had no significant correlation with the GTV of pathology or tumor diameter.

The simplest method, which is widely used, is a visual interpretation of the PET scan and definition of contours as judged visually in cooperation with an experienced nuclear medicine physician (7-9). Another method using SUV is absolute SUV and regression function or source-to-background ratio. Hatt et al. (10) established the repeatability and reproducibility limits of several volume-related PET image-derived indices-namely tumor volume, mean SUV, total glycolytic volume, and total proliferative volume. Fixed and adaptive thresholding, fuzzy C-means, and fuzzy locally adaptive Bayesian (FLAB) methodology were considered for tumor volume delineation. The reproducibility of different quantitative parameters associated with functional volumes depends significantly on the delineation approach. State-of-the-art algorithms for functional volume segmentation use adaptive thresholding. The new 3-FLAB algorithm is able to extract the overall tumor from the background tissues and delineate variable-uptake regions within the tumors, with improved accuracy and robustness compared with adaptive threshold (tumor and background intensities) and fuzzy C-means. The gradientbased segmentation method applied to denoised and deblurred images proved to be more accurate than the source-to-background ratio method (5).

The different techniques to define tumor contour by ${ }^{18} \mathrm{~F}-\mathrm{FDG}$ PET in radiotherapy planning resulted in substantially different volumes, especially in patients with inhomogeneous tumors (4). In our study, manual contouring was preferred to autocontouring at a $50 \%$ threshold for PET tumor volume delineation (1). However, manual delineation of functional volumes using PET images leads to high inter- and intraobserver variability (11). Furthermore, manual contouring is a long process when it has to be performed in 3 dimensions (12). As for the conclusion in our paper, when using autocontouring of the target in NSCLC, one should consider manual contouring of ${ }^{18} \mathrm{~F}-\mathrm{FDG}$ PET to check for any missed disease that might be incompletely covered (1).

We agree with the recommendation of Hatt and colleagues that future studies investigating this issue should include a more accurate methodology, such as a segmentation algorithm. We also need to attain more data on functional volume compared with pathologic volume. Much more work must be done to resolve these issues concerning the delineation target of NSCLC using $\mathrm{PET} / \mathrm{CT}$, and we still must correlate with the gold standardpathologic findings—-whenever possible.

\section{REFERENCES}

1. Wu K, Ung YC, Hwang D, et al. Autocontouring and manual contouring: which is the better method for target delineation using ${ }^{18} \mathrm{~F}-\mathrm{FDG}$ PET/CT in non-small cell lung cancer? J Nucl Med. 2010;51:1517-1523.

2. Wu K, Ung YC, Hornby J, et al. Thresholds for radiotherapy target definition in non-small-cell lung cancer: how close are we to the pathologic findings? Int J Radiat Oncol Biol Phys. 2010;77:699-706.

3. Hatt M, Turzo A, Roux C, et al. A fuzzy Bayesian locally adaptive segmentation approach for volume determination in PET. IEEE Trans Med Imaging. 2009;28: 881-893.

4. Nestle U, Kremp S, Schaefer-Schuler A, et al. Comparison of different methods for delineation of ${ }^{18}$ F-FDG PET-positive tissue for target volume definition in radiotherapy of patients with non-small cell lung cancer. $J$ Nucl Med. 2005;46: 1342-1348.

5. Geets X, Lee JA, Bol A, et al. A gradient-based method for segmenting FDG-PET images: methodology and validation. Eur J Nucl Mol Imaging. 2007;34:1427-1438.

6. Yu J, Li X, Xing L, et al. Comparison of tumor volumes as determined by pathologic examination and FDG-PET/CT images of non-small cell lung cancer: a pilot study. Int J Radiat Oncol Biol Phys. 2009;75:1468-1474.

7. Nestle U, Walter $\mathrm{K}$, Schmidt $\mathrm{S}$, et al. ${ }^{18} \mathrm{~F}$-deoxyglucose positron emission tomography (FDG-PET) for the planning of radiotherapy in lung cancer: high impact in patients with atelectasis. Int J Radiat Oncol Biol Phys. 1999;44:593-597.

8. Kiffer JD, Berlangieri SU, Scott AM, et al. The contribution of ${ }^{18} \mathrm{~F}-$ fluoro-2deoxy-glucose positron emission tomographic imaging to radiotherapy planning in lung cancer. Lung Cancer. 1998;19:167-177.

9. Nestle U, Hellwig D, Schmidt S, et al. 2-Deoxy-2-[ $\left.{ }^{18} \mathrm{~F}\right]$ fluoro-D-glucose positron emission tomography in target volume definition for radiotherapy of patients with non-small-cell lung cancer. Mol Imaging Biol. 2002;4:257-263.

10. Hatt M, Cheze Le Rest C, Aboagye EO, et al. Reproducibility of ${ }^{18}$ F-FDG and ${ }^{18}$ F-FLT PET tumor volume measurements. J Nucl Med. 2010;51:1368-1376.

11. Krak NC, Boellaard R, Hoekstra OS, et al. Effects of ROI definition and reconstruction method on quantitative outcome and applicability in a response monitoring trial. Eur J Nucl Med Mol Imaging. 2005;32:294-301.

12. Hatt M, Cheze le Rest C, Descourt P, et al. Accurate automatic delineation of heterogeneous functional volumes in positron emission tomography for oncology applications. Int J Radiat Oncol Biol Phys. 2010;77:301-308.

\section{Kailiang Wu}

Yee C. Ung*

*Odette Cancer Centre

2075 Bayview Ave.

Toronto, Ontario M4N 3M5, Canada

E-mail: yee.ung@sunnybrook.ca

DOI: 10.2967/jnumed.110.085399 